\title{
The conduction properties of $\alpha, \omega$-diaminoalkanes and hydrazine bridging gold electrodes
}

\author{
Sören Wohlthat ${ }^{\text {a }}$, Fabian Pauly ${ }^{\mathrm{b}, \mathrm{c}}$, Jeffrey R. Reimers ${ }^{\mathrm{a}, *}$ \\ ${ }^{a}$ School of Chemistry, The University of Sydney, Sydney, New South Wales 2006, Australia \\ ${ }^{\mathrm{b}}$ Institut für Theoretische Festkörperphysik and DFG-Center for Functional Nanostructures, Universität Karlsruhe, \\ 76128 Karlsruhe, Germany \\ ${ }^{\mathrm{C}}$ Institut für Nanotechnologie, Forschungszentrum Karlsruhe, 76021 Karlsruhe, Germany
}

\begin{abstract}
The conduction properties of $\alpha, \omega$-diaminoalkanes and hydrazine bridging gold electrodes are investigated using density functional theory in combination with Green's function techniques and group theory. We show that more than $99 \%$ of the current at low bias is carried by one channel of $A^{\prime}$ symmetry whose form is invariant to both the alkane chain length and realistic changes of the injection energy. The total conductance decreases exponentially with a decay constant of $0.73 \AA^{-1}$ with increasing chain length while the calculated gold-amine contact resistance is ca. $150 \mathrm{k} \Omega$.
\end{abstract}

The development of molecular electronics requires understanding of electron transport between conductors through single molecules $[1,2]$. Due to the availability of different bonding mechanisms, bonding sites, and anchoring groups, the electrical properties of the metal-molecule-metal junctions can vary considerably [3]. For gold electrodes, studies often use thiols as anchoring group to chemisorbe organic molecules to the metal [4-20]. However, recently there is a trend toward amine anchoring groups to establish contacts, with these providing improved reliability and reproducibility of conductance measurements [21-27].

A commonly used class of organic molecules bridging the gold electrodes is the alkanes. These have large HOMO-LUMO gaps $(8-11 \mathrm{eV})$ and the Fermi energy of gold $(-5.0 \mathrm{eV})[28]$ lies well inside this energy gap. Therefore, the conduction mechanism is off-resonant tunneling, where the zero-bias conductance $G$ decreases exponentially with the length $d$ of the molecular junction $[2,6]$ :

\footnotetext{
* Corresponding author. Fax: +61 293513329.

E-mail address: reimers@chem.usyd.edu.au (J.R. Reimers).
}

$G=G_{\mathrm{c}} \exp (-\beta d)$.

where $1 / G_{\mathrm{c}}$ is the junction contact resistance and $\beta$ is the inverse decay length, given usually per $\AA$ or per number of methylene groups.

The exponential decay of the conductivity of alkane$\alpha, \omega$-dithiols bond to gold electrodes via the sulfur linkages has been thoroughly studied with various experimental and theoretical methods reporting inverse decay lengths between 0.5 and 1.1 per methylene group [4-13,20]. However, a few experiments using amines instead have also been reported $[23,24]$. The following two values have recently been measured for the inverse decay length of gold- $\alpha, \omega$-diaminoalkane-gold junctions: 0.81 by Chen et al. [23] and 0.91 by Venkataraman et al. [24] per methylene group. Despite the success of these experiments, theoretical investigations are sparse. Venkataraman et al. [24] estimated the inverse decay length from the frontier orbital splitting to be 0.94 per methylene group, while Fagas and Greer using the program VICI [29] that relies on the maximum entropy principle with application of open system boundary conditions through use of the Wigner function, reported a $\beta$-value of 0.98 per methylene [30]. They also 
predicted the bond through an $\mathrm{NH}_{2}$ group to be more stable than that through a dehydrogenated NH group.

To investigate the transport properties of alkanes further, we study gold- $\alpha, \omega$-diaminoalkane-gold junctions using the more commonly used approach of density functional theory (DFT) in combination with Green's function techniques. The alkanes studied have a length of $N=1-10$ methylene units, and the gold-hydrazine-gold junction containing $N=0$ methylene units is also considered. We emphasise the junction resistance and examine the properties of the conductance channels and their symmetry [31]. Details of the available channels can in principle be measured in shot noise experiments and therefore provides a valuable piece of information for comparisons between experiment and theory [32-34].

The DFT calculations are performed using TURBOMOLE v5.7 [35] with a split valence polarization basis set for all non-hydrogen atoms [36] and the BP86 exchange-correlation functional $[37,38]$. First, we optimize the geometry for the $\alpha, \omega$-diaminoalkanes and the hydrazine, where both amine groups are bonded to a single gold atom, in their respective symmetry $\left(C_{2 v}\right.$ for an odd number of methylene units and $C_{2 h}$ for an even number and for hydrazine). Our geometries are in agreement with the ones obtained by Fagas and Greer [30]. The calculated $\mathrm{Au}-\mathrm{N}$ bond lengths are between $2.37 \AA$ and $2.38 \AA$ and the $\mathrm{C}-\mathrm{N}$ bond lengths vary between $1.46 \AA$ and $1.48 \AA$; the $\mathrm{Au}-\mathrm{N}$ bond length decreases and the $\mathrm{C}-\mathrm{N}$ bond length grows with an increasing number of methylene units. As an exception, the step from 1,2-diaminoethane to 1,3-diaminopropane is reversed to this trend. After this optimization, the bridging molecules are connected to two gold fec pyramids oriented in the (111)-direction, so that the symmetry remains $C_{2 h}$ or $C_{2 v}$, respectively (see example structures in Fig. 1). The pyramids consist of four layers, with 1, 3, 6, and 10 atoms in the layers from the inside to the outside. The electronic structures are then obtained to an accuracy better than $10^{-6}$ Hartree in the total energy. To calculate the conduc- tance in this open system, we divide it into three parts (left $(\mathrm{L})$, extended-molecule $(\mathrm{M})$, and right $(\mathrm{R})$ ), where the extended-molecule part consists of the molecule and the inner two layers of each pyramid. The conductance is then ascertained using Green's function techniques [28,39,40]. One requirement for this method to be valid is that the overlap and Hamiltonian matrix elements coupling the left and right side vanish. Here, these elements of the overlap are always smaller than $10^{-7}$ and the ones of the Hamiltonian smaller than $10^{-7}$ Hartree.

Results for zero-bias conductance obtained from the transmission at the Fermi energy are shown in a semi-logarithmic plot in Fig. 2 as a function of the length $N$ of the junction. Also shown in this figure are the low bias conductances measured by Venkataraman et al. [24] and Chen et al. [23]. In absolute magnitude, our calculated values are very similar to those observed by Chen et al., these being ca. twice those measured by Venkataraman et al.; however, the absolute accuracy expected from these calculations is insufficient to discriminate between the two sets of experimental results. An exponential fit to the calculated data for $N=1-10$ methylene gives an inverse decay length of $\beta=0.93$ per methylene (equivalent to $0.73 \AA^{-1}$ ), also in good agreement with the experimental results of $0.81 \pm$ 0.01 [23] and $0.91 \pm 0.03$ [24] as well as with the calculated result of 0.98 by Fagas and Greer [30]. Interestingly, extrapolation of our calculated results for the diaminoalkanes yields a contact resistance $1 / G_{\mathrm{c}}$ of two amine-Au bonds as $\sim 150 \mathrm{k} \Omega$, close to the calculated value of $116 \mathrm{k} \Omega$ for the $\mathrm{Au}$-hydrazine-Au junction. This indicates that the alkanes act purely as spacers, meaning that they do not have a strong influence on the electronic structure of the Au-amine interface.

We also investigate the symmetry of conduction. The conductance point group is the geometric point group of the junction reduced by its end-to-end symmetry [31]. Therefore, the conductance point group of all junctions investigated here is $C_{s}$; the mirror plane is shown in red
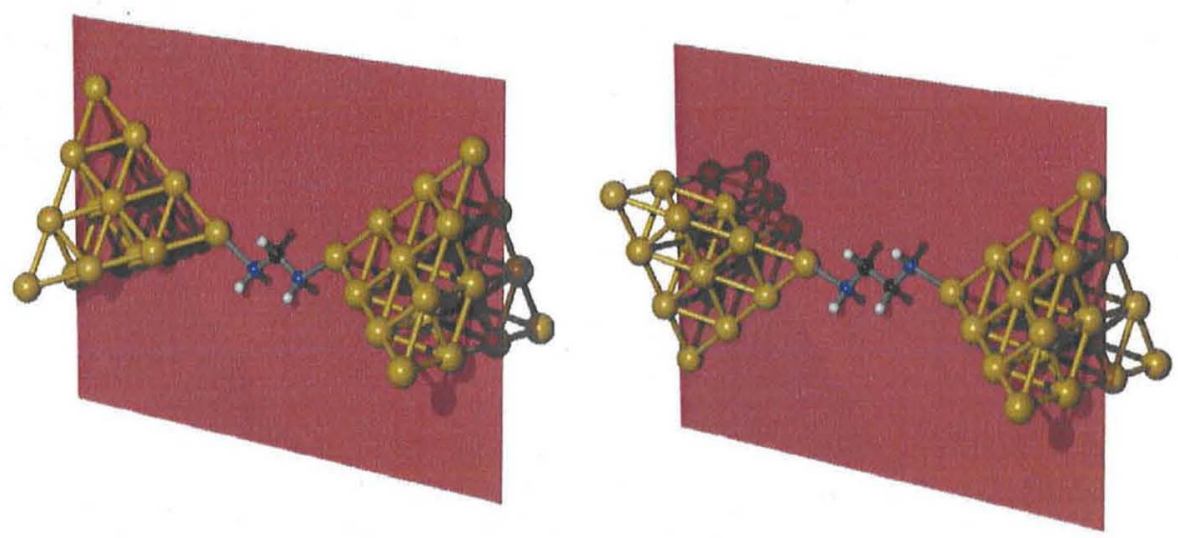

Fig. 1. Example geometries of $\mathrm{Au}-\alpha,()$-diaminoalkane- $\mathrm{Au}$ junctions: (left) $\mathrm{Au}_{20}$-diaminomethane- $\mathrm{Au}_{20}$ in $C_{2 v}$ symmetry. (right) $\mathrm{Au} \mathrm{u}_{20}-1,2-\mathrm{diaminoe}-$ thane- $\mathrm{Au}_{20}$ in $C_{2 h}$ symmetry. The symmetry element of the $C_{s}$ conductance point group is the red plane. The nitrogen atoms are represented in blue, the carbon atoms in black, the gold atoms in yellow, and the hydrogen atoms in grey. (For interpretation of the references to color in this figure legend, the reader is referred to the web version of this article.) 


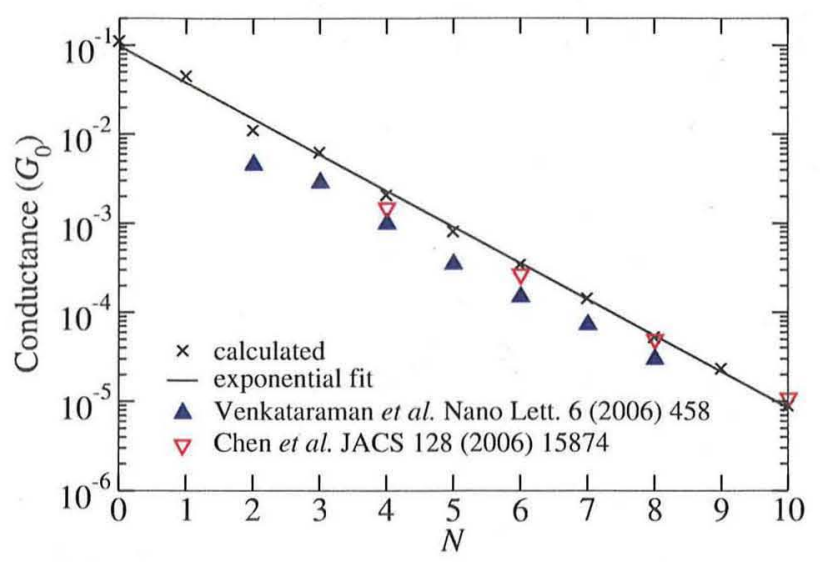

Fig. 2. Total conductance as a function of the alkane chain length $N$ of the $\mathrm{Au}-\alpha,(\mathrm{w})$-diaminoalkane-Au junctions. Black crosses-calculated, black line-exponential fit to $\alpha, \omega$-diaminoalkanes with 1-10 methylene units, red triangles-observed by Chen et al. [23], blue triangles-Venkataraman et al. [24]. (For interpretation of the references to color in this figure legend, the reader is referred to the web version of this article.)

in Fig. 1. While we concentrate here on highly-symmetric structures that appear somewhat artificial, use of asymmetric electrode-molecule junctions does not influence the qualitative picture as the symmetry of the conduction channels is essentially a molecular property [31]; in addition, the symmetric molecular geometries are close to the high-conductance conformations [41] probably present at low temperature experiments. To determine the symmetry species of the conductance channels, we extend the program of [39], adding a similarity transformation $s$ to the right of the transmission matrix $[42,43]$

$t=\Gamma_{\mathrm{R}}^{1 / 2} G_{\mathrm{MM}}^{\mathrm{a}} \Gamma_{\mathrm{L}}^{1 / 2}$,

to bring $t^{\dagger} t$ to bock diagonal form in the symmetry-adapted basis. In Eq. (2), $\Gamma$ are twice the imaginary part of the selfenergy matrices of the left or right side and $G_{\mathrm{MM}}^{\mathrm{a}}$ is the advanced Green's function of the extended-molecule. In this way, we obtain the following expression for the conductance:

$G=G_{0} \operatorname{Tr}\left[s^{\dagger} t^{\dagger} t s\right]$

where $G_{0}=2 e^{2} / h$ is the so-called quantum of conductance. The conductance channels are the orthogonal eigenvectors of the Hermitian matrix $t^{\dagger} t$. We define the matrix $C$, whose column vectors consist of the coefficients of the eigenvectors in the atomic orbital basis, from

$\left[s^{\dagger} t^{\dagger} t s\right] C=C T$

where $T$ is the diagonal matrix of eigenvalues.

As an example, we plot the transmission as a function of energy for $\mathrm{Au}$-diaminomethane-Au in Fig. 3. The total transmission (sum of the eigenvalues $T_{n}$ of $t^{\dagger} t$ ) is rather flat around the Fermi energy, producing as a first approximation a linear $I-V$ curve, in agreement with the experimental results of [23]. Furthermore, we track the channels adiabat-

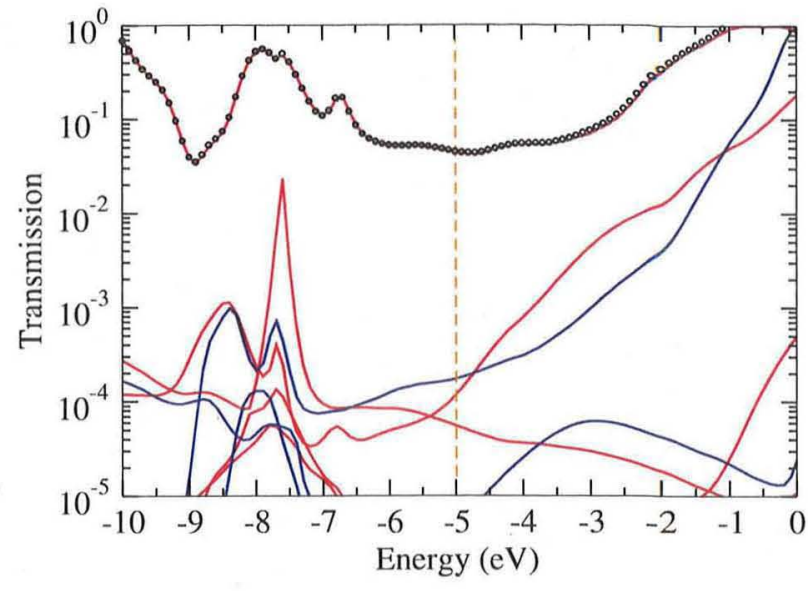

Fig. 3. Transmission as a function of energy for $\mathrm{Au}$-diaminomethane-Au in $C_{s}$ conductance symmetry. The black circles are the total transmission while the red lines show the eigenvalues $T_{n}$ corresponding to channels of $A^{\prime}$ symmetry and the blue lines similarly refer to the channels of $A^{\prime \prime}$ symmetry. (For interpretation of the references to color in this figure legend, the reader is referred to the web version of this article.)

ically as a function of energy, identifying eigenvalues at sequential energies as belonging to a conserved conductance channel if their coefficient vectors have a squared absolute value of their scalar product larger than 0.5 . The results indicate that the transmission is dominated by just one channel of $A^{\prime}$ symmetry. However, other weakly conducting channels of both $A^{\prime}$ and $A^{\prime \prime}$ symmetry are also apparent (see Fig. 3). Independent of the length of the molecular methylene chain, one single channel carries more than $99 \%$ of the current at low bias.

To understand the nature of the dominant $A^{\prime}$ conduction channel, its form is monitored diabatically as a function of the length of the molecular bridge. In detail, we project the coefficient vectors of each junction onto the orbitals of the gold and nitrogen atoms and compare them to a once chosen basis vector. This basis vector is represented in Fig. 4 where it is seen to be localized on the atoms near the interface of the extended-molecule with the L electrode. As the vector does not delocalize significantly onto the organic bridging molecule, its form is independent of the length of

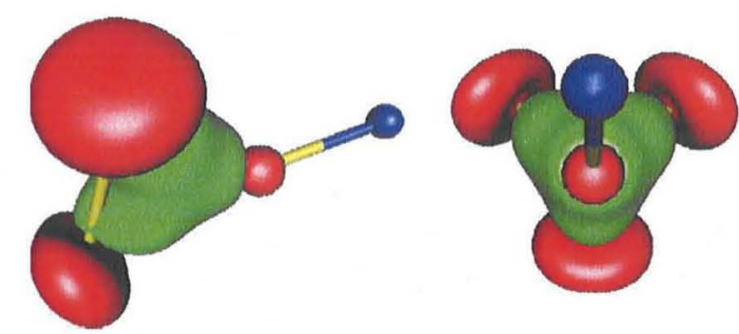

Fig. 4. Representation (green and red) of the orbital contributions of the inner four gold atoms of the $\mathrm{L}$ electrode and its chemisorbed nitrogen atom to the first conductance channel in $A^{\prime}$ symmetry. Gold is represented in yellow and nitrogen in blue. (For interpretation of the references to color in this figure legend, the reader is referred to the web version of this article.) 
the bridge. The selection of the orbitals at the $\mathrm{L}$ interface over those at the $\mathrm{R}$ interface occurs as the transmission is written as the trace of $t^{\dagger} t=\Gamma_{\mathrm{L}}^{1 / 2} G_{\mathrm{MM}}^{r} \Gamma_{\mathrm{R}} G_{\mathrm{MM}}^{a} \Gamma_{\mathrm{L}}^{1 / 2}$ instead of say the equivalent form $t t^{\dagger}=\Gamma_{\mathrm{R}}^{1 / 2} G_{\mathrm{MM}}^{\mathrm{a}} \Gamma_{\mathrm{L}} G_{\mathrm{MM}}^{r} \Gamma_{\mathrm{R}}^{1 / 2}$. In this way, the eigenvectors tell the orbitals involved in transmission through the chosen electrode to extended-molecule interface. As shown in the figure, the coupling to the $\mathrm{L}$ electrode is dominated by the orbitals on the gold atoms in the extended-molecule that connect to it. This has the pseudo$C_{3 v}$ symmetry of the electrodes rather than the symmetry of the organic molecule.

Conduction channels provide simple non-interfering representations of the physical process that result in the through-molecule conductance, but they are not uniquely defined. The dominant eigenvector of the Hermitian matrix $t^{\dagger} t$ considered herein has distinct physical significance as its form changes only slowly as continuous variables such as the injection energy are varied. Further, it also remains invariant to changes in discrete variables such as the molecular chain length. Closely related, commonly considered $[34,44,45]$ alternative channels include those obtained by applying the method developed by Büttiker $[46,47]$ to the basic conduction matrix $\Gamma_{\mathrm{L}} G_{\mathrm{MM}}^{r} \Gamma_{\mathrm{R}} G_{\mathrm{MM}}^{\mathrm{a}}$, producing the same eigenvalues as obtained by diagonalizing $t^{\dagger} t$ akin to Eq. (4) but different coefficient vectors $C^{\prime}$ related by the transformation $C^{\prime}=\Gamma_{\mathrm{L}}^{1 / 2} C$. As $\Gamma_{\mathrm{L}}^{1 / 2}$ is a near singular matrix [34], orthogonality of the eigenvectors is destroyed by this process and hence these eigenvectors change erratically and discontinuously in response to small physical perturbations [34]. Diagonalization [42] of $t^{\dagger} t$ thus presents a much more effective means for obtaining physical information concerning the nature of conductance channels.

Nevertheless, the eigenvectors $C$ provide information only about the junction between the electrodes and the extended-molecule, and while this location may be varied to good effect, Cuevas et al. [44] have shown that it is the properties of the narrowest neck in the conduction pathway that dictates the number and nature of the conduction channels. While separation of the electrodes and extended-molecule at this neck leads to a simple understanding as to why just one channel dominates the conduction $[34,48,49]$, it does not lead to converged numerical simulations of the conductance at, e.g. the level of DFT. Here, by including various layers of gold atoms in the extended-molecule so that no assumptions are made as to the location of the narrowest neck, we show using accurate simulations that this neck takes the same form for hydrazine as it does for long diaminoalkanes, hence demonstrating that it is located at the chemisorption site(s).

In summary, we have shown that the Green's function theory of single-molecule conduction combined with DFT to model the electronic structure provides results for conduction through gold- $\alpha, \omega$-diaminoalkane-gold junctions in good agreement with both experiment and alternative computational strategies. Further, we showed that the conductance is dominated at all chain lengths by transport through a single channel of $A^{\prime}$ symmetry. The nature of this channel examined on a plane just inside the electrode tip showed the pseudo- $C_{3 v}$ symmetry of the tip, while the narrowest path of the channel is shown to be located at the chemisorptions sites.

\section{Acknowledgements}

S.W. and J.R.R. would like to acknowled ge the Australian Partnership for Advanced Computing for computing resources and the Australian Research Council for funding. F.P. acknowledges the funding of a Young Investigator Group at KIT.

\section{References}

[1] A. Nitzan, M.A. Ratner, Science 300 (2003) 1384.

[2] N.J. Tao, Nature Nanotechnol. 1 (2006) 173.

[3] Y.Q. Xue, M.A. Ratner, Phys. Rev. B 69 (2004) 085403.

[4] V.B. Engelkes, J.M. Beebe, C.D. Frisbie, J. Am. Chem. Soc. 126 (2004) 14287.

[5] J.K. Tomfohr, O.F. Sankey, Phys. Rev. B 65 (2002) 245105.

[6] M.H. Lee, G. Speyer, O.F. Sankey, Phys. Status Solidi B 243 (2006) 2021 .

[7] K. Weber, L. Hockett, S. Creager, J. Phys. Chem. B 101 (1997) 8286.

[8] A. Salomon, D. Cahen, S. Lindsay, J. Tomfohr, V.B. Engelkes, C.D. Frisbie, Adv. Mater. 15 (2003) 1881.

[9] B.Q. Xu, N.J.J. Tao, Science 301 (2003) 1221

[10] G. Fagas, P. Delaney, J.C. Greer, Phys. Rev. B.73 (2006) 241314(R).

[11] X.L. Li, J. He, J. Hihath, B.Q. Xu, S.M. Lindsay, N.J. Tao, J. Am. Chem. Soc. 128 (2006) 2135.

[12] W. Haiss, R.J. Nichols, H. van Zalinge, S.J. Higgins, D. Bethell, D.J. Schiffrin, Phys. Chem. Chem. Phys. 6 (2004) 4330.

[13] X.D. Cui et al., J. Phys. Chem. B 106 (2002) 8609.

[14] M.A. Reed, C. Zhou, C.J. Muller, T.P. Burgin, J.M. Tour, Science 278 (1997) 252.

[15] M. Elbing et al., Proc. Natl. Acad. Sci. USA 102 (2005) 8815.

[16] J.K. Viljas, F. Pauly, J.C. Cuevas, Phys. Rev. B 76 (2007) 033403.

[17] G.C. Solomon, A. Gagliardi, A. Pecchia, T. Frauenheim, A. Di Carlo, J.R. Reimers, N.S. Hush, J. Chem. Phys. 124 (2006) 094704.

[18] K.H. Müller, Phys. Rev. B 73 (2006) 045403.

[19] F. Pauly, J.K. Viljas, J.C. Cuevas, <arxiv:/cond-mat0709.3588>, 2007.

[20] W.Y. Wang, T.H. Lee, M.A. Reed, Proc. IEEE 93 (2005) 1815.

[21] C.W. Chu, J.S. Na, G.N. Parsons, J. Am. Chem. Soc. 129 (2007) 2287.

[22] J. Herrmann, K.-H. Müller, G. Wei, D. Bray, M. Roberts, B. Raguse, L. Wieczorek, IEEE Proc. ICONN 2006 (2006) 314.

[23] F. Chen, X.L. Li, J. Hihath, Z.F. Huang, N.J. Tao, J. Am. Chem. Soc. 128 (2006) 15874.

[24] L. Venkataraman, J.E. Klare, I.W. Tam, C. Nuckolls, M.S. Hybertsen, M.L. Steigerwald, Nano Lett. 6 (2006) 458.

[25] L. Venkataraman, J.E. Klare, C. Nuckolls, M.S. Hybertsen, M.L. Steigerwald, Nature 442 (2006) 904

[26] J.R. Quinn, F.W. Foss, L. Venkataraman, M.S. Hybertsen, R. Breslow, J. Am. Chem. Soc. 129 (2007) 6714.

[27] S.Y. Quek, L. Venkataraman, H.J. Choi, S.G. Louie, M.S. Hybertsen, J.B. Neaton, Nano Lett. 7 (2007) 3477.

[28] F. Pauly, J.K. Viljas, J.C. Cuevas, G. Schön, <arxiv:/condmat0705.3285>, 2007.

[29] P. Delaney, J.C. Greer, Int. J. Quantum Chem. 100 (2004) 1163.

[30] G. Fagas, J.C. Greer, Nanotechnology 18 (2007) 424010.

[31] G.C. Solomon, A. Gagliardi, A. Pecchia, T. Frauenheim, A. Di Carlo, J.R. Reimers, N.S. Hush, J. Chem. Phys. 125 (2006) 184702.

[32] D. Djukic, J.M. van Ruitenbeek, Nano Lett. 6 (2006) 789. 
[33] R. Cron, M.F. Goffman, D. Esteve, C. Urbina, Phys. Rev. Lett. 86 (2001) 4104.

[34] G.C. Solomon, A. Gagliardi, A. Pecchia, T. Frauenheim, A. Di Carlo, J.R. Reimers, N.S. Hush, Nano Lett. 6 (2006) 2431.

[35] R. Ahlrichs, M. Bär, M. Häser, H. Horn, C. Kölmel, Chem. Phys. Lett. 162 (1989) 165

[36] A. Schäfer, H. Horn, R. Ahlrichs, J. Chem. Phys. 97 (1992) 2571.

[37] J.P. Perdew, Phys. Rev. B 33 (1986) 8822.

[38] A.D. Becke, Phys. Rev. A 38 (1988) 3098.

[39] F. Pauly, Ph.D., Universität Karlsruhe (TH), Karlsruhe, 2007.

[40] S. Wohlthat, F. Pauly, J.K. Viljas, J.C. Cuevas, G. Schön, Phys. Rev. B 76 (2007) 075413.

[41] D.R. Jones, A. Troisi, J. Phys. Chem. C 111 (2007) 14567.
[42] J. Heurich, J.C. Cuevas, W. Wenzel, G. Schon, Phys, Rev. Lett. 88 (2002) 256803

[43] A. Bagrets, N. Papanikolaou, I. Mertig, Phys. Rev. B 75 (2007) 235448.

[44] J.C. Cuevas, A.L. Yeyati, A. Martin-Rodero, Phys. Rev. Lett. 80 (1998) 1066.

[45] D. Jacob, J.J. Palacios, Phys. Rev. B 73 (2006) 075429.

[46] M. Büttiker, IBM J. Res. Dev. 32 (1988) 63.

[47] M. Brandbyge, M.R. Sorensen, K.W. Jacobsen, Phys. Rev. B 56 (1997) 14956.

[48] M. Kemp, A. Roitberg, V. Mujica, T. Wanta, M.A. Ratner, J. Phys Chem. 100 (1996) 8349.

[49] A. Troisi, A. Ratner Mark, Phys. Rev. B 72 (2005) 033408. 